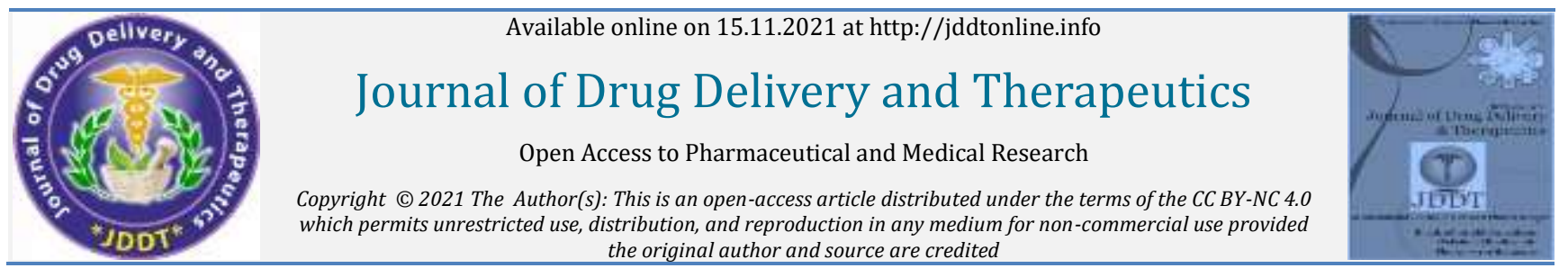

Open Access Full Text Article the original author and source are credited

Review Article

\title{
A review on medicinal plants as potential sources of natural immunomodulatory action
}

\author{
Omji Porwal*, Mehmet Ozdemir, Duran Kala, Esra Tariq Anwer \\ Department of Pharmacognosy, Faculty of pharmacy, Tishk International University-Erbil, Kurdistan Region, Iraq
}

Article Info:

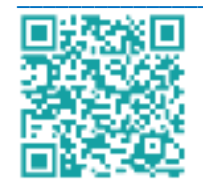

\section{Article History:}

Received 02 October 2021 Reviewed 06 November 2021 Accepted 09 November 2021 Published 15 November 2021

\section{Cite this article as:}

Porwal 0, Ozdemir M, Kala D, Anwer ET, A review on medicinal plants as potential sources of natural immunomodulatory action, Journal of Drug Delivery and Therapeutics. 2021; 11(6):324-331

DOI: http://dx.doi.org/10.22270/jddt.v11i6.5125

\section{*Address for Correspondence:}

Dr. Omji Porwal, Professor (Full), Faculty of Pharmacy, Tishk International University, $100 \mathrm{mt}$. Street, near Filkey Baz (Square), across Qazi Muhammad, 44001, Erbil, KRG/IRAQ

\section{Abstract}

The concept of immunomodulation was proposed by Edward Jenner, while working on polio vaccine in 1796. A brawny, fine-functioning immune system is the keystone of excellent health. Immune replies are the consequence of an effectual interaction among innate (natural and non-specific) and acquired (adaptive and specific) components of the immune system. Inequity or failure of the immune systems is connected with a variety of chronic illness counting allergies, autoimmune diseases, cancers and furthers. Diverse innate and adaptive immune cells that are incorporated in this multifaceted networking organization may symbolize talented targets for expanding immunotherapeutics for treating specific immune illness. An assorted array of natural, synthetic, and recombinant compounds is accessible with both advantages and demerits. A range of phytochemicals have been remote, differentiated and customized for expansion and employ as avoidance or cure of human diseases, but the request of customary or novel medicinal plants for employ as immunomodulators in indulgencing immune diseases is still comparatively limited. At present, there is much-growing interest in the use of medicinal plants as modulators of the complex immune system. Numerous therapeutic consequences of plant extracts have been recommended to be because of their extensive assortment of immunomodulatory effects and persuade on the immune system of the human body. In present review paper, various medicinal plants, their resultant crude or fractionated phyto extracts and the precise phytochemicals remote from them are conversed in terms of their immunomodulatory bioactivities. We also review their possible for future expansion as immunomodulatory or inflammation-regulatory therapeutics or agents.

Keywords: Immunomodulation, Immune system, Phytochemicals, Medicinal plant, Plant extracts

\section{Introduction}

It is an evident from the human history that medicinal plants have been the treatment regimen to cure a variety of diseases, including diseases origins by bacteria, fungi, insects, and viruses. The consequences revealed by the plants are because of the chemicals there in them and they toil in the similar way as the conventional drugs. Though, there are equally possibilities for these plants to have several prospective injurious and poisonous consequences too. These undesired side consequences can be abridged by processing of the plant's crude result ${ }^{1}$. A moment ago, the elevated thought of plant-based natural products is salaried by researchers because of many unfavorable side effects of contemporary salutary medicines. Also, the synthetic drugs are extra pricey to get in association with herbal products. Huge quantity of chemical entities with a variety of pharmacological activities created from plants is helpful to encourage human fitness. In 2013, 1453 novel chemical entities patented from natural products accepted by US Food and Drug Administration ${ }^{2}$. They have been widely used as health supplements, nutritive products, medications since prehistoric times. Natural product-based drugs promote a significant role of the pharmaceutical industry. Recently, secure drugs with little side consequences and elevated discriminating ligands that work on sole disease target become a assignment of drug development programs ${ }^{3}$. Immunity is the body's capability to recognize and repel/fight against wide range of diseases and also possibly against toxic microorganisms.The immune system maintains homeostasis within the body in a normal condition of host. Assorted endogenous and exogenous agents contribute to the competence and purpose of the immune system that guide to the immunosuppression or immunostimulation 4 . The alteration of host's immune system contributes to the progression of abnormal conditions such as cancer. Therefore, the intonation of host's immune reply to raise the capability of this system in eliminating the aetiological 
agents that caused diseases might reduce this trouble ${ }^{5}$ Immunomodulation could be defined as the modification process of immune reactions that function to regulate immune responsiveness to treat illnesses ${ }^{6}$. The request of immunomodulators for either as a treatment or avoidance of a variety of illness that connected with malfunctioning of immune reactions became the main thought 7 . The main purposes of immunomodulators are either to suppress immune responses as a treatment for autoimmune diseases or to enhance them that applied in immunodeficiency and infectious diseases ${ }^{8}$. Additionally, the usage of immunostimulant agents also essentially acts as an adjuvant to chemotherapy for various illnesses ${ }^{9}$. These compounds may get affected by some factors like dose, route of administration, timing of administration and also site of $\operatorname{action}^{10}$.

\section{Immunity and classification of immunomodulators}

Immunity is the mainly significant essential fundamental construction of the body; to be precise it is a complex system with multiple shields. The chief shield/barricade is: The skin; here hotness and $\mathrm{pH}$ of body there may be inappropriate for obtainable requirement. albeit, the microbes go into the body, they are provoked by acquired or innate immune systems ${ }^{11}$. Innate immunity is one of the chief immune protections, in answer to foreign and dangerous objects. Innate immunity is an immune protection mechanism that is set with receptors, for immediate reply. Here, there is no need of any genetic recombination or any other process ${ }^{12}$. Acquired immunity known as adaptive immunity. It is mostly observed in blood, in tissue juices, or co-joined to cell surface. Immunoglobulin also known as the antibody receptor is the chief key as antigen-specific receptor in the shape of ' $Y$ ' 13 .

The biomolecules of artificial or biological source able of suppressing, modulating, and stimulating each feature of immune system with innate and adaptive immune system are recognized as immunorestoratives, immunomodulators, immunoaugmentors, or biological response modifiers. Scientifically, Immunomodulators are usually classified into immunostimulants, immunoadjuvants, and immunosuppressants in clinical practice ${ }^{14}$.

Immunoadjuvants-These are utilized to improve the effectiveness of preparations/shots and thus are deemed as immune stimulants. They are regarded as genuine modulators of the immune system. It is noticed quite often that they are manipulated as selectors among cellular and humoral assistant/helper T1 (TH1) and helper T2cells (TH2), immune-protective, immunedestructive and immunoglobulin $\mathrm{E}$ vs. immunoglobulin $\mathrm{G}$ (IgE vs. IgG) reactions, an immense task for the vaccines/shot designers ${ }^{15}$.

$>$ Immunostimulants-These are foreseen as improvements of a body's opposition towards infection which is integrally not precise. They have ability to function by adaptive or innate immune reaction. As they increase the body's fundamental level of immune these are recognized as immunopotentiators and promoter agents ${ }^{16}$.

$>$ Immunosuppressants-These are considered as intrinsically and operationally heterogenous class of drugs, concurrently dispensed in an amalgamation so as to cure several sorts of tissue/organ transplant dismissal and autoimmune disorder ${ }^{17}$. Furthermore, these agents can also be employed in the cure of infection connected hypersensitivity reactions, immunopathology, and autoimmune illness. A quantity of monoclonal antibodies and chemically created compounds are also being employed as immunomodulators. Consequently, immunomodulatory entities with extra security and efficiency are still in require. Because of the incidence of chemical drugs-related unfavorable results, natural immunomodulators are the possible agents to restore them in therapeutic schedules.

Screening methods for immunomodulatory property

In-vitro and animal models are used to test the effectiveness and toxicity of the active constituents, which are separated and extracted from a plant extract that illustrates the bioactivities. Numerous in vitro and in vivo techniques of pharmacological screening of therapeutic plants having immunomodulatory activity have been reported ${ }^{18}$.

\section{In vitro methods}

$>$ Inhibition of histamine discharge from mast cells

> Mitogens persuaded lymphocyte proliferation

$>$ Inhibition of $\mathrm{T}$ cell proliferation

$>$ Chemiluminescence in macrophages

$>$ Inhibition of dihydroorotate dehydrogenase

$>$ Plaque forming colony

\section{In vivo methods}

$>$ Spontaneous autoimmune diseases in animals

$>$ Passive cutaneous anaphylaxis

$>$ Acute systemic anaphylaxis in rats

$>$ Anti-anaphylactic activity (Schultz-Dale reaction)

$>$ Delayed-type hypersensitivity

$>$ Reversed passive Arthus reaction

$>$ Arthus type immediate hypersensitivity

$>$ Adjuvant arthritis in rats

$>$ Proteoglycan-induced progressive polyarthritis in mice

$>$ Experimental autoimmune thyroiditis

$>$ Coxsackievirus B3-induced myocarditis

$>$ Collagen type II-induced arthritis in rats

$>$ Porcine cardiac myosin-induced autoimmune myocarditis in rats

$>$ Experimental allergic encephalomyelitis

$>$ Inhibition of allogeneic transplant rejection

$>$ Acute graft versus host disease in rats

$>$ Influence on SLE-like disorder in MRL/lpr mice

$>$ Prevention of experimentally induced myasthenia gravis in rats

$>$ Glomerulonephritis induced by anti-basement membrane antibody in rats

$>$ Autoimmune uveitis in rats ${ }^{19}$.

\section{Plant derived immunomodulators}

Currently, mainstream of investigate and expansion motionless centers on biochemical's, biologics or sole complexes as guide compounds that aspire at exacting targets connected with a illness. It is tricky to achieve solo 
complex chemicals with elevated selectivity and effectiveness and short toxicity for targeted molecular/cellular targets and illness. Therefore, the design and expansion of drug applicants from several conservative or complementary and substitute medicines is expanding attention. The avoidance and cure of illness using plantsupported medicines has been accounted in human history. In every culture and through all ages dissimilar parts of an enormous quantity of plants were employed as drugs against every type of ailments. Vincristine vinblastine, and their semi-synthetic imitative isolated from the Catharanthus roseus paclitaxel from Taxus brevifolia, capsaicin from Capsicum species, and galantamine from Galanthus caucasicus are instances of medicines based on plant complexes. The plant-based complexes that provided as lead structures and/or were chemically altered are morphine (scores of derivatives), dicoumarol (warfarin), camptothecin (topotecan and irinotecan), artemisinin (artemether), and salicylic acid (acetylsalicylic acid) ${ }^{20}$. The immunomodulatory traits of plant-based therapeutics have assembled thought of researchers ${ }^{21}$. Innovative expertise's and the extreme research on immunomodulatory natural products, their extracts, plants, and their lively moieties with immunomodulatory possible, may give us with precious entities to expand as new immunomodulatory agents to addition the current chemotherapies. This reviewfocuses on the various plants have immunomodulatoryactivity. Several herbs have potent immunomodulatory action was mention in Table 1.

Table 1List of medicinal plants having immunomodulatory activity ${ }^{22-103}$

\begin{tabular}{|c|c|c|c|}
\hline Plant name & Parts used & Chemical constituent & Model used \\
\hline Allium sativum & Bulbs & Allicin & Hemagglutination \\
\hline Adhatoda vasica & Leaves & Quinazoline, vasicinone, essential oils & Neutrophil adhesion, DTH \\
\hline Abrus precatorius & Seeds & Alkaloids, phenolics, tannins, saponins & HA titer, DTH response, PI \\
\hline Abutilon indicum & Whole plant & Flavonoids, triterpenoids & $\begin{array}{l}\text { HA titer, DTH response, neutrophil } \\
\text { adhesion test, and carbon clearance } \\
\text { test }\end{array}$ \\
\hline Argyreia speciosa & Roots & Glycosides & $\begin{array}{l}\text { Cellular, humoral immunity, DTH } \\
\text { reaction }\end{array}$ \\
\hline Asparagus racemosus & Roots & Saponins, sitosterols & SRBC-sensitized animals \\
\hline Andrographis paniculata & Leaves & Diterpenes & DTH mouse model \\
\hline $\begin{array}{l}\text { Acanthopanax } \\
\text { sessiliflorus }\end{array}$ & Shoots, roots & Biopolymers & Lymphocyte-proliferating effects \\
\hline Acacia catechu & Leaf & Tannins, flavonoids & $\begin{array}{l}\text { Neutrophil adhesion, carbon clearance } \\
\text { test }\end{array}$ \\
\hline Artemisia annua & Herb & Artemisinin & DTH, lymphocytic proliferation assay \\
\hline Achillea millefolium & Leaves & Flavonoids, alkaloids, coumarins & DTH, hemagglutination \\
\hline Aloe vera & $\begin{array}{l}\text { Gel from } \\
\text { leaves }\end{array}$ & Anthraquinone glycosides & Hematological, serological studies \\
\hline Aesculus indica & Leaf & Alkaloids, saponins, tannins & Neutrophil index, neutrophil adhesion \\
\hline Azadirachta indica & Flowers & Azadirachtin & $\begin{array}{l}\text { Antibody titer phagocytic activity, nitro } \\
\text { blue tetrazolium dye, DTH reaction }\end{array}$ \\
\hline Bauhinia variegata & Root, bark & Flavonoids, $\beta$-sitosterol, lupeol & Human neutrophils \\
\hline Boerhaavia diffusa & Herb & Alkaloids & Circulating antibody titer \\
\hline Balanitesrox burghii & Leaf & Alkaloids, flavonoids, tannins, saponins & $\begin{array}{l}\text { Carbon clearance test, serum } \\
\text { immunoglobulin }\end{array}$ \\
\hline Tridax procumbens & Aerial parts & Tannins, flavonoids, alkaloids, steroids & DTH model \\
\hline Urena lobata & Fruits & Flavonoids, glycosides & Phagocytic activity \\
\hline Withania somnifera & Root & Withanolides & Bone marrow cellularity \\
\hline $\begin{array}{l}\text { Chlorophytum } \\
\text { borivilianum }\end{array}$ & Roots & Polysaccharides & $\begin{array}{l}\text { Phagocytosis using carbon clearance } \\
\text { method }\end{array}$ \\
\hline Cleome gynandra & $\begin{array}{l}\text { Leaf, seeds, } \\
\text { roots }\end{array}$ & Hexacosanol, kaempferol & $\begin{array}{l}\text { Carbon clearance test, DTH, antibody } \\
\text { titer }\end{array}$ \\
\hline Calendula officinalis & Flowers & Polysaccharides, proteins, fatty acids & Phagocytosis \\
\hline Centella asiatica & Herb & Triterpenoid saponins & Cell-mediated, humoral immune \\
\hline
\end{tabular}




\begin{tabular}{|c|c|c|c|}
\hline & & & responses \\
\hline Clitoria ternatea & Aerial parts & $\beta$-sitosterol, kaempferol & $\begin{array}{l}\text { DTH, antibody, drug-induced } \\
\text { myelosuppression }\end{array}$ \\
\hline Citrus aurantifolia & Fruits & Volatile oils & $\begin{array}{l}\text { Cell proliferation assay, } \\
\text { immunoblotting }\end{array}$ \\
\hline Capparis zeylanica & Leaf & Flavonoids & Phagocytosis, delayed hypersensitivity \\
\hline Curcuma longa & Rhizome & Curcumin & Humoral antibody response to SRBC \\
\hline Cleome gynandra & Aerial parts & $\begin{array}{l}\text { Flavonoids, alkaloids, terpenoids, } \\
\text { steroids }\end{array}$ & $\begin{array}{l}\text { Carbon clearance method, } \\
\text { cell-mediated immunity, } \\
\text { immunostimulatory }\end{array}$ \\
\hline Eclipta alba & Leaves & Triterpenoid glycosides & Phagocytic index antibody titer \\
\hline Euphorbia hirta & Herb & Quercitol, myricitrin, gallic acid & Phagocytic index \\
\hline Ficus carica & Leaf & $\begin{array}{l}\text { Phenolic compound, phytosterol, } \\
\text { volatile oils }\end{array}$ & $\begin{array}{l}\text { Cellular immune response, humoral } \\
\text { antibody response }\end{array}$ \\
\hline Cissampelos pareira & Roots & Alkaloids & Humoral antibody titer \\
\hline Caesalpinia bonducella & Seeds & $\begin{array}{l}\text { Flavonoids, alkaloids, tannins, amino } \\
\text { acids }\end{array}$ & Neutrophil adhesion test, HA \\
\hline Ficus benghalensis & Root & Alkaloids, steroids, flavonoids, tannins & $\begin{array}{l}\text { Hypersensitivity, hemagglutination } \\
\text { reactions. }\end{array}$ \\
\hline Ganoderma lucidum & Whole plant & $\begin{array}{l}\text { Flavonoids, triterpenes, } \\
\text { polysaccharides }\end{array}$ & Proliferation of lymphocytes \\
\hline Gymnema sylvestre & Leaves & Alkaloids, tannins, flavonoids & Neutrophil locomotion, chemotaxis test \\
\hline Nyctanthes arbortristis & Leaf & Iridoid glucosides & Humoral immunity, DTH \\
\hline Murraya koenigii & Leaves & $\begin{array}{l}\text { Coumarins, carbazole alkaloids, } \\
\text { glucoside }\end{array}$ & $\begin{array}{l}\text { PI, nitric acid assay, humoral antibody, } \\
\text { DTH reaction }\end{array}$ \\
\hline Mangifera indica & Stem bark & Alkaloids, tannins, flavonoids & Humoral antibody response to SRBC \\
\hline Moringa oleifera & Leaves & Vitamin A, B, C, carotenoids, saponins & DTH test neutrophil adhesion, HA \\
\hline Morus alba & $\begin{array}{l}\text { Fruits, leaves, } \\
\text { bark }\end{array}$ & Flavonoids, anthocyanins & $\begin{array}{l}\text { Humoral immunity, serum } \\
\text { immunoglobulin }\end{array}$ \\
\hline Habenaria intermedia & Tubers & Alkaloids phenolic compounds & $\begin{array}{l}\text { DTH test, carbon clearance test for } \\
\text { phagocytic activity }\end{array}$ \\
\hline Hyptis suaveolens & Leaf, flowers & Lupeol, $\beta$-sitosterol & $\begin{array}{l}\text { Humoral immune response, lipid } \\
\text { peroxide enzyme }\end{array}$ \\
\hline Hibiscus rosa-sinensis & Flowers & $\begin{array}{l}\text { Alkaloids, flavonoids, terpenoids, } \\
\text { tannins }\end{array}$ & $\begin{array}{l}\text { Carbon clearance method, } \\
\text { cell-mediated immunity, } \\
\text { immunostimulatory }\end{array}$ \\
\hline Lycium barbarum & Fruits & Polysaccharide-protein complexes & HA PI lymphocytic proliferation \\
\hline Salicornia herbacea & Herb & Polysaccharides & Phagocytic activity on opsonized \\
\hline Syzygium cumini & Seeds & $\begin{array}{l}\text { Alkaloids, flavonoids, glycosides, } \\
\text { phytosterols }\end{array}$ & $\begin{array}{l}\text { Carbon clearance method, } \\
\text { hemagglutination titer, DTH }\end{array}$ \\
\hline Panax ginseng & Fruits, root & $\begin{array}{l}\text { Ginsenosides, panaxdiol, panaxtriole, } \\
\text { oleanolic acid }\end{array}$ & $\begin{array}{l}\text { Antibody plaque-forming cell response, } \\
\text { circulating antibody titer against sheep } \\
\text { erythrocytes }\end{array}$ \\
\hline Salacia chinensis & Roots & $\begin{array}{l}\text { Flavonoids, tannins, alkaloids, } \\
\text { carbohydrates }\end{array}$ & HA titer, DTH response \\
\hline Silybum marianum & Flowers & Flavonoids & Macrophage migration index \\
\hline Ocimum sanctum & Entire plant & $\begin{array}{l}\text { Essential oils such as eugenol, } \\
\text { carvacrol, derivatives of ursolic acid, } \\
\text { apigenin }\end{array}$ & $\begin{array}{l}\text { Enhance the production of } \mathrm{RBC}, \mathrm{WBC} \text {, } \\
\text { hemoglobin }\end{array}$ \\
\hline Picrorhiza kurroa & Leaf & Alkaloids, flavonoids, tannins, saponins & Cell-mediated, humoral components \\
\hline
\end{tabular}




\begin{tabular}{|l|l|l|l|}
\hline Piper longum & Fruits & Alkaloids & HA, PI, macrophage migration index \\
\hline Terminalia arjuna & Leaves, bark & $\begin{array}{l}\text { Flavonoids, } \\
\text { oligomericproanthocyanidins, tannins }\end{array}$ & Hemagglutination \\
\hline Tinospora cordifolia & Entire herb & Alkaloids & DTH, bone marrow cellularity \\
\hline Trapa bispinosa & Fruits & Flavonoids, proteins, carbohydrates & Neutrophils, hemagglutination titer \\
\hline
\end{tabular}

DTH: Delayed-type hypersensitivity, RBC: Red blood cell, WBC: White blood cell, SRBC: Sheep red blood cell, HA: Hemagglutination antibody, PI: Phagocytic index

\section{Future Prospects}

From ancient times plant derived medicines and folklore medicines have been employed for the drug design and development of therapeutic agents ${ }^{104}$. Herbal and traditional botanical products are good alternatives to conventional chemotherapy 105 . Currently researchers are enthralled towards plant gained therapeutics and the research is based on study for a few plant biochemicals in the type of the sole complex as lead molecule concerned with particular target linked with disease ${ }^{106}$. Several plant obtained complexes have been recognized over the years which possess immunomodulatory traits but the appropriate, efficient, and multidisciplinary approach is requisite for picking out active constituents from different medicinal plants and their different medicinal effects using modern techniques ${ }^{107}$. Two approaches can be followed for developing successful drugs from medicinal plants. First one is the phytochemical approach, which depends on identifying the active principle and developing pure phytochemicals as drugs. Yet this type of drug discovery is costly and also time consuming. The second approach is a phytotherapeutic approach wherein standardized crude drug preparations can be employed as drugs with modern standards of protection and efficiency. As far as the Indian therapeutic plants are concerned, the second approach could be followed.

\section{Conclusion}

Immunomodulation employed medicinal plants can give alternative to conservative chemotherapy for a diversity of illness, particularly when host protection mechanism has to be stimulated under the situations of impairedimmune response or when a discriminating immunosuppression is preferred insituations like autoimmune disorders. There is great potential for the detection of further precise immunomodulators which mimic or antagonize the biological effects of cytokines and interleukins, and the refinement of assays for these mediators will create specific and sensitive screens. Naturalremedies should be revisited as important sources of novel ligands capable of targeting specific cellular receptors.

\section{Funding}

There was no financial support.

\section{Conflict of interest}

None

\section{References}

1. Sharma P, Kumar P, Sharma R, Gupta G, Chaudhary A. Immunomodulators: Role of medicinal plants in immune system. Natl J Physiol Pharm Pharmacol 2017; 7(6):552-556. https://doi.org/10.5455/njppp.2017.7.0203808032017

2. Katz L, Baltz RH. Natural product discovery: past, present, and future. J. Ind. Microbiol. Biotechnol 2016; 43(2-3): 155-176. https://doi.org/10.1007/s10295-015-1723-5
3. David B, Wolfender JL, Dias DA. The pharmaceutical industry and natural products: Historical status and new trends. Phytochem Rev 2015; 14 (2): 299-315. https://doi.org/10.1007/s11101014-9367-z

4. Jantan I, Ahmad W, Bukhari SNA. Plant-derived immunomodulators: An insight on their preclinical evaluation and clinical trials. Frontier Plant Sci 2015; 6:655. https://doi.org/10.3389/fpls.2015.00655

5. Razali FN, Sinniah SK, Hussin H, Zainal Abidin N, Shuib AS. Tumor suppression effect of Solanum nigrum polysaccharide fraction on breast cancer via immunomodulation. Int J Biol Macromol 2016; 92:185-193. https://doi.org/10.1016/j.ijbiomac.2016.06.079

6. Rasheed HMF, Rasheed F, Qureshi AW, Jabeen Q. Immunostimulant activities of the aqueous methanolic extract of Leptadeniapyrotechnica, a plant from Cholistan desert. J Ethnopharmacol 2016; 186:244-250. https://doi.org/10.1016/j.jep.2016.03.039

7. Sharma KR, Adhikari A, Jabeen A, Dastagir N, Kalauni SK, Choudhary M, et al., Immunomodulatory studies on triterpenoids from Scopari adulcis Linn. Biochem Pharmacol 2015; 4 (4):182. https://doi.org/10.4172/2167-0501.1000182

8. Ilyas U, Katare DP, Aeri V, Naseef PP. A review on hepatoprotective and immunomodulatory herbal plants. Pharmacognosy Rev 2016; 10 (19):66-70. https://doi.org/10.4103/09737847.176544

9. Pujol J, Vansteenkiste J, Martino T, Atanackovic D, Reck M, Thomeer M et al., Safety and immunogenicity of MAGE-A3 cancer immunotherapeutic with or without adjuvant chemotherapy in patients with resected stage IB to III MAGE-A3positive non-small-cell lung cancer. J Thoracic Oncol 2015; 10 (10):1458-1467. https://doi.org/10.1097/JT0.0000000000000653

10. Tzianabos AO. Polysaccharide immunomodulators as therapeutic structural aspects and biologic function. Clin Microbiol Rev 2000; 13(4):523-533. https://doi.org/10.1128/CMR.13.4.523

11. Turvey SE, Broide DH. Chapter 2 -innate immunity. J Aller Clin Immunol 2010; 125 (2):S24-S32. https://doi.org/10.1016/j.jaci.2009.07.016

12. Nicoholson BL. The immune system. Essays in Biochem 2016; 60 (3):275-301. https://doi.org/10.1042/EBC20160017

13. Kumar D, Arya V, Kaur R, Ali Bhat Z, GuptaVK, Kumar V. A review of immunomodulators in the Indian traditional health care system. J Microbiol, Immunol Infection 2012; 45 (3):165-184. https://doi.org/10.1016/j.jmii.2011.09.030

14. Billiau A, Matthys P. Modes of action of freund's adjuvants in experimental modes of autoimmune disease. J Leukoc Biol 2001; $70(6): 849-860$.

15. Gertsch J, Viveros-Paredes JM, Taylor P. Plant immunestimulantsscientific paradigm or myth. J Ethnopharmacol 2011; 136 (3):385-391. https://doi.org/10.1016/j.jep.2010.06.044

16. George A, Shah PA, Shrivastav PS. Guar gum: Versatile natural polymer for drug delivery applications. Eur Polym J 2018; 112:722-735. https://doi.org/10.1016/j.eurpolymj.2018.10.042

17. Narayanaswamy V. Origin and development of ayurveda. Anc Sci life $1981 ; 1(1): 1-7$. 
18. Vogel HG. Drug Discovery and Evaluations. 2nd ed. New-York: Springer-Verlang; 2002. pp. 775-90. https://doi.org/10.1007/3540-29837-1_10

19. Mohan MR, Rao GBS, Narender B, Kumar CA, Rao PV, Bakshi V. Indian medicinal plants used as immunomodulatory agents: A review. Int J Green Pharm 2019; 13 (4):312-318.

20. Oberlies NH, Kroll DJ. Camptothecin and taxol: historic achievements in natural products research. J Nat Prod 2004; 67:129-135. https://doi.org/10.1021/np030498t

21. Jantan I, Ahmad W, Bukhari SNA. Plant-derived immunomodulators: an insight on their preclinical evaluation and clinical trials. Front Plant Sci 2015; 6: 655. https://doi.org/10.3389/fpls.2015.00655

22. Singh N, Tailang M, Mehta SC. A review on herbal plants as immunomodulators. Int J Pharm Sci Res 2016; 7(9):3602-3610.

23. Nagarathna PKM, Reena K, Reddy S, Wesley J. Review on immunomodulation and immunomodulatory activity of some herbal plants. Int J Pharm Sci Rev Res 2013; 22(1):223-230.

24. Patel R, Gautam P, Tabish M, Kumar S. Herbal plants used for immunomodulatory action: a review. Int J Res Pharm Sci 2012; 2(3):14-26. https://doi.org/10.7439/ijpp.v2i2.388

25. Ismail S, Asad M. Immunomodulatory activity of Acacia catechu. Indian J Physiol Pharmacol 2009; 53:25-33.

26. Jeong SC, Jeong YT, Yang BK, Song CH. Chemical characteristics and immuno-stimulating properties of biopolymers extracted from Acanthopanaxsessiliflorus. J Biochem Mol Biol 2006; 39:84-90. https://doi.org/10.5483/BMBRep.2006.39.1.084

27. Noori S, Naderi GA, Hassan ZM, Habibi Z, Bathaie SZ, Hashemi SM et al., Immunosuppressive activity of a molecule isolated from Artemisia annuaon DTH responses compared with cyclosporin A. Int Immunopharmacol 2004;4:1301-6. https://doi.org/10.1016/j.intimp.2004.05.003

28. Sharififar F, Pournourmohammadi S, Arabnejad M. Immunomodulatory activity of aqueous extract of Achillea wilhelmsii C. Koch in mice. Indian J Exp Biol 2009; 47:668-71.

29. Mukesh SS, Patil MB, Sharma S, Bhat V. Aloe vera: Plant of immortality. Int J Pharm Sci Res 2010; 1:7-10.

30. Porwal O, Singh SK, Patel DK, Gupta S, Tripathi R, Katekhaye S. Cultivation, Collection and Processing of Medicinal Plants. Bioactive Phytochemicals: Drug Discovery to Product Development. 2020:14-30. https://doi.org/10.2174/9789811464485120010005

31. Ramanunny AK, Wadhwa S, Gulati M, Gupta S, Porwal O, Jha NK et al., Development and validation of RP-HPLC method for $1^{\prime}$ Acetoxychavicol acetate (ACA) and its application in optimizing the yield of ACA during its isolation from Alpiniagalanga extract as well as its quantification in nanoemulsion. South African J Botany 2021 https://doi.org/10.1016/j.sajb.2021.10.012

32. Clement F, Pramod SN, Venkatesh YP. Identity of the immunomodulatory proteins from garlic (Allium sativum) with the major garlic lectins or agglutinins. Int Immunopharmacol2010; 10:316-24. https://doi.org/10.1016/j.intimp.2009.12.002

33. Chakraborthy GS. Evaluation of immunomodulatory activity of Aesculus indica. Int J Pharm Tech Res 2009; 1:132-4.

34. Naik SR, Hule A. Evaluation of immunomodulatory activity of an extract of andrographolides from Andographis paniculata. Planta Med 2009; 75:785-91. https://doi.org/10.1055/s-00291185398

35. Bopana N, Saxena S. Asparagus racemosus ethnopharmacological evaluation and conservation needs. J Ethnopharmacol 2007; 110:1-15. https://doi.org/10.1016/j.jep.2007.01.001

36. Sood A, Kumar B, Singh SK, Prashar P, Gautam A, Gulati M, et al., Flavonoids as potential therapeutic agents for the management of diabetic neuropathy. Curr Pharm Design. 2020; 26(42):54685487. https://doi.org/10.2174/1381612826666200826164322
37. Singh S, Singh SK, Kumar B, Kaur B, Khursheed R, Gulati M, et al., Effect of co-administration of herbal extracts with copper nanoparticles: a novel two-pronged approach in treating type 2 diabetes. Recent Innov Chem Eng.2020; 13(5):366-378. https://doi.org/10.2174/2405520413999200719140819

38. Banerjee M, Khursheed R, Yadav AK, Singh SK, Gulati M, Pandey DK, et al., A systematic review on synthetic drugs and phytopharmaceuticals used to manage diabetes. Curr Diabetes Rev 2020; 16(4):340-356. https://doi.org/10.2174/1573399815666190822165141

39. Gokhale AB, Damre AS, Saraf MN. Investigations into the immunomodulatory activity of Argyreia speciosa. J Ethnopharmacol 2003; 84:109-14. https://doi.org/10.1016/S0378-8741(02)00168-X

40. Dashputre NL, Naikwade NS. Immunomodulatory activity of Abutilon indicum Linn. On albino mice. Int J Pharm Sci Res 2010; 1:178-84.

41. Tilwari A, Shukla NP, Pathirissery UD. Immunomodulatory activity of the aqueous extract of seeds of Abrus precatorius Linn (Jequirity) in mice. Iran J Immunol2011; 8:96-103.

42. Vinothapooshan G, Sundar K. Immunomodulatory activity of various extracts of Adhatoda vasica Linn. In experimental rats. Afr J Pharm Pharmacol 2011; 5:306-10. https://doi.org/10.5897/AJPP10.126

43. Shah AS, Gunjal MA, Juvekar AR. Immunomostimulatory activity of aqueous extract of Azadirachtaindicaflowers on specific and non specific immune response. J Nat Rem 2009; 9:35-42.

44. Ghaisas MM, Saikh SA, Deshpande AD. Evaluation of immunomodulatory activity of ethanolic extract of stem bark of Bauhinia variegata Linn. Int J Green Pharm 2009; 3:70-4. https://doi.org/10.4103/0973-8258.49379

45. Mungantiwar AA, Nair AM, Shinde UA, Dikshit VI, Saraf MN, Thakur VS, et al., Studies on the immunomodulatory effects of Boerhaavia diffusa alkaloidal fraction. J Ethnopharmacol1999; 65:125-31. https://doi.org/10.1016/S0378-8741(98)00153-6

46. Korek J, Shete RV, Kabra MP, Rachhadiya RM, Attal AR. Immunomodulatory activity of Balanite roxburghii. J Pharm Res Health Care 2014; 3:63-7.

47. Hussain FHS, Amin HIM, Patel DK, Porwal O. An overview of the therapeutic potential of Iris persica. Curr Tradit Med 2021 $7(2): 152-160$.

https://doi.org/10.2174/2215083806666200117111320

48. Porwal O, Gupta S, Nanjan MJ, Singh A. Classical taxonomy studies of medicinally important Ipomoea leari. Ancient Sci Life 2015; 35(1):34. https://doi.org/10.4103/0257-7941.165628

49. Porwal O, Nanjan MJ, Chandrasekar MJN, Srinivasan R, Gupta S. Anticancer potential of solanum jasminoides Int J Pharm Sci Res 2014;5(9):3768-3774

50. Chandrasekar MJN, Srinivasan R, Porwal O, Nanjan MJ. In-Vitro antioxidant activity of solanum jasminoides paxt extracts. J Nat Remed 2012; 12(2):115-118.

51. Thakur M, Bhargava S, Dixit VK. Immunomodulatory activity of Chlorophytum borivilianum Sant. F. Evid Based Complement Alternat Med 2007; 4:419-23. https://doi.org/10.1093/ecam/nel094

52. Gaur K, Kori ML, Nema RK. Comparative screening of immunomodulatory activity of hydro-alcoholic extract of Hibiscus rosa-sinensis Linn. and ethanolic extract of Cleome gynandra Linn. Glob J Pharmacol 2009; 3:85-9.

53. Jiménez-Medina E, Garcia-Lora A, Paco L, Algarra I, Collado A, Garrido F, et al., A new extract of the plant Calendula officinalis produces a dual in vitro effect: Cytotoxic anti-tumor activity and lymphocyte activation. BMC Cancer 2006; 6:119. https://doi.org/10.1186/1471-2407-6-119

54. Mali RJ, Hatapakki BC. An in vitro study of effect of Centella asiatica on phagocytosis by human neutrophils. Int J Pharm Sci 
Nanotechnol 2008; 1:297-302.

https://doi.org/10.37285/ijpsn.2008.1.3.13

55. Anarthe SJ, Spoorthisudha P, Swetha M, Raju MG. In vitro and in vivo screening of Clitoria ternatea (Linn.) for immunomodulatory activity. Int J Chem Tech Res 2017; 10:11625.

56. Antony S, Kuttan R, Kuttan G. Immunomodulatory activity of curcumin. Immunol Invest 1999; 28:291-303. https://doi.org/10.3109/08820139909062263

57. Vishwas S, Gulati M, Kapoor B, Gupta S, Singh SK, Awasthi A, et al., Expanding the arsenal against Huntington's disease-Herbal drugs and their nanoformulations. Curr Neuropharmacol 2021; 19(7):957-989.

https://doi.org/10.2174/1570159X18666201109090824

58. Malviya R, Sundram S, Fuloria S, Subramaniyan V, Sathasivam KV, Azad AK, et al., Evaluation and characterization of tamarind gum polysaccharide: The biopolymer. Polymers 2021; 13(18):3023. https://doi.org/10.3390/polym13183023

59. Porwal O, Malviya R, Ameen MSM, Anwar ET, Sharma A. A review on effect of various parameters on the rheological behaviour, thermal properties and viscosity of potato starch. Curr Materials Sci 2021; E-pub Ahead of Print. https://doi.org/10.2174/2666145414666210521214130

60. Malviya R, Raj S, Fuloria S, Subramaniyan V, Sathasivam K, Kumari U, et al., Evaluation of antitumor efficacy of chitosantamarind gum polysaccharide polyelectrolyte complex stabilized nanoparticles of simvastatin. Int J Nanomed 2021; 16:25332553. https://doi.org/10.2147/IJN.S300991

61. Gharagozloo M, Ghaderi A. Immunomodulatory effect of concentrated lime juice extract on activated human mononuclear cells. J Ethnopharmacol2001; 77:85-90. https://doi.org/10.1016/S0378-8741(01)00269-0

62. Surendra SA, Saurabh CK, Gokul ST. Studies on immunomodulatory Activity of Capparis zeylanica leaf extracts. Int J Pharm Sci Nanotechnol2010; 3:887-92. https://doi.org/10.37285/ijpsn.2010.3.1.14

63. Bafna A, Mishra S. Antioxidant and immunomodulatory activity of the alkaloidal fraction of Cissampelos pareira Linn. Sci Pharm 2010; 78:21-31. https://doi.org/10.3797/scipharm.0904-16

64. Shukla S, Mehta A, John J, Mehta P, Vyas SP, Shukla S, et al., Immunomodulatory activities of the ethanolic extract of Caesalpinia bonducella seeds. J Ethnopharmacol2009; 125:2526. https://doi.org/10.1016/j.jep.2009.07.002

65. Kaur G, Singh SK, Kumar R, Kumar B, Kumari Y, Gulati M, et al. Development of modified apple polysaccharide capped silver nanoparticles loaded with mesalamine for effective treatment of ulcerative colitis. J Drug Del Sci Tech 2020; 60: 1-10. https://doi.org/10.1016/j.jddst.2020.101980

66. Som S, Singh SK, Khatik GL, Kapoor B, Gulati M, Kuppusamy G, et al., Quality by design-based crystallization of curcumin using liquid antisolvent precipitation: micromeritic,

biopharmaceutical, and stability aspects. Assay Drug Develop Techn 2020; 18(1):11-33.

https://doi.org/10.1089/adt.2018.913

67. Singh A, Porwal O, Sharma N, Singh A, Kumar S, Sharma PK. Effects of prebiotics on git and human health: a review. J Pure Appl Microbiol 2007; 1(1):69-82.

68. Anwar ET, Gupta N, Porwal O, Sharma A, Malviya R, Singh A, et al., Skin diseases and their treatment strategies in sub-saharan african regions. Infect Disord Drug Targets. 2021. E-pub Ahead of Print.

https://doi.org/10.2174/1871526521666210927120334

69. Ameen MS, Malviya R, Porwal O, Anwar ET, Pant S, Sharma A. Novel Strategies and Model Studies for Colon Targeted Drug Delivery. Drug Delivery Letters. 2021; 11(2):156-63. https://doi.org/10.2174/2210303111666210118141406

70. Jayathirtha MG, Mishra SH. Preliminary immunomodulatory activities of methanol extracts of Eclipta alba and Centella asiatica. Phytomedicine 2004; 11:361-5.

https://doi.org/10.1078/0944711041495236

71. Patil SB, Naikwade NS, Magdum CS. Review on phytochemistry and pharmacological aspects of Euphorbia hirta Linn. J Pharm Res Health Care 2009; 1:113-33.

72. Vikas VP, Shandavi CB, Vijay RP. Studies on immunomodulatory activity of Ficuscarica. Int J Pharm PharmSci 2010; 2:97-9.

73. Gabhe SY, Tatke PA, Khan TA. Evaluation of the immunomodulatory activity of the methanol extract of Ficusbenghalensisroots in rats. Indian J Pharmacol2006; 38:2715. https://doi.org/10.4103/0253-7613.27024

74. Habijanic J, Berovic M, Wraber B, Hodzar D, Boh B. Immunostimulatory effects of fungal polysaccharides from Ganoderma lucidum sub merged biomass cultivation. Food TechnolBiotechnol 2001; 39:327-31.

75. Sudhakar K, Fuloria S, Subramaniyan V, Sathasivam KV, Azad AK, Swain SS, et al., Ultraflexible liposome nanocargo as a dermal and transdermal drug delivery system. Nanomaterials 2021; 11(10):2557. https://doi.org/10.3390/nano11102557

76. Khan TA, Azad AK, Fuloria S, Nawaz A, Subramaniyan V, Akhlaq $\mathrm{M}$, et al., Chitosan-coated 5-fluorouracil incorporated emulsions as transdermal drug delivery matrices. Polymers 2021;13(19):3345. https://doi.org/10.3390/polym13193345

77. Malviya R, Jha S, Fuloria NK, Subramaniyan V, Chakravarthi S, Sathasivam K, et al., Determination of temperature-dependent coefficients of viscosity and surface tension of tamarind seeds (tamarindus indica ) polymer. Polymers 2021; 13(4):610. https://doi.org/10.3390/polym13040610

78. Jha R, Singh A, Sharma PK, Porwal O, Fuloria NK. Graphene-based nanomaterial system: A boon in the era of smart nanocarriers. J Pharm Investig 2021; 51:245-280.

https://doi.org/10.1007/s40005-021-00513-3

79. Ghosh D, Singh SK, Khursheed R, Pandey NK, Kumar B, Kumar R, et al., Impact of solidification on micromeritic properties and dissolution rate of self-nanoemulsifying delivery system loaded with docosahexaenoic acid. Drug Develop Indus Pharm 2020; 46(4):597-605. https://doi.org/10.1080/03639045.2020.1742143

80. Kumari Y, Kaur G, Kumar R, Singh SK, Gulati M, Khursheed R, et al., Gold nanoparticles: New routes across old boundaries. Adv Colloid interface Sci 2019; 274:102037. https://doi.org/10.1016/j.cis.2019.102037

81. Malik JK, Manvi FV, Nanjwade BK, Alagawadi KR, Sinsh S Immunomodulatory activity of Gymnema sylvestre R. Br. leaves on in vitro human neutrophils. J Pharm Res 2009; 2:1284-6. https://doi.org/10.4103/0973-8258.41184

82. Sahu MS, Sahu RA, Verma A. Immunomodulatory activity of alcoholic extract of Habenaria intermedia in mice. Int J Pharm Pharm Sci2013; 5:406-9.

83. Jain V, Bhagwat D, Jat RC, Bhardwaj S. The immunomodulation potential of Hyptis suaveolens. Int J Pharm Res Dev 2005; 1:1-6.

84. Gan L, Zhang SH, Liu Q, Xu HB. A polysaccharide-protein complex from Lycium barbarum upregulates cytokine expression in human peripheral blood mononuclear cells. Eur J Pharmacol 2003; 471:217-22. https://doi.org/10.1016/S00142999(03)01827-2

85. Gupta A, Gautam MK, Singh RK, Kumar MV, Rao CV, Goel RK, et al., Immunomodulatory effect of Moringa oleifera Lam. Extract on cyclophosphamide induced toxicity in mice. Indian J Exp Biol2010; 48:1157-60.

86. Bharani SE, Asad M, Dhamanigi SS, Chandrakala GK. Immunomodulatory activity of methanolic extract of Morus alba Linn. (mulberry) leaves. Pak J Pharm Sci 2010; 23:63-8.

87. Shah AS, Wakade AS, Juvekar AR. Immunomodulatory activity of methanolic extract of Murraya koenigii (L) spreng. Leaves. Indian J Exp Biol2008; 46:505-9. 
88. Makare N, Bodhankar S, Rangari V. Immunomodulatory activity of alcoholic extract of Mangifera indica L. In mice. J Ethnopharmacol 2001; 78:133-7. https://doi.org/10.1016/S0378-8741(01)00326-9

89. Kannan M, Ranjit SA, Ajith KT, Jegatheswari P, Subburayalu S. Studies on immuno-bioactivities of Nyctanthes arbortristis(Oleaceae). Afr J Microbiol Res 2007; 1:88-91. https://doi.org/10.1007/978-0-387-70638-2_1074

90. Caroline JR, Rama V, Rameshkumar G. Immunomodulatory activity of aqueous extract of Ocimum sanctum in rat. Int J Pharm Biomed Res 2011; 233-8.

91. Sharma ML, Rao CS, Duda PL. Immunostimulatory activity of Picrorhiza kurrooa leaf extract. J Ethnopharmacol 1994; 41:18592. https://doi.org/10.1016/0378-8741(94)90031-0

92. Sunila ES, Kuttan G. Immunomodulatory and antitumor activity of Piper longum Linn. And piperine. J Ethnopharmacol 2004; 90:339-46. https://doi.org/10.1016/j.jep.2003.10.016

93. Kim MH, Byon YY, Ko EJ, Song JY, Yun YS, Shin T, et al. Immunomodulatory activity of ginsan, a polysaccharide of panax ginseng, on dendritic cells. Korean J Physiol Pharmacol 2009; 13:169-73. https://doi.org/10.4196/kjpp.2009.13.3.169

94. Meeran SM, Katiyar S, Elmets CA, Katiyar SK. Silymarin inhibits UV radiation-induced immunosuppression through augmentation of interleukin-12 in mice. Mol Cancer Ther2006 5:1660-8. https://doi.org/10.1158/1535-7163.MCT-06-0095

95. Im SA, Kim K, Lee CK. Immunomodulatory activity of polysaccharides isolated from Salicornia herbacea. IntImmunopharmacol 2006; 6:1451-8. https://doi.org/10.1016/j.intimp.2006.04.011

96. Mastan SK, Saraseeruha A, Gourishankar V. Immunomodulatory activity of methanolic extract of Syzygium cumini seeds. Pharmacologyonline 2008; 3:895-903.

97. Gohla SH, Zeman RA, Bogel M, Jurkiewicz E, Schrum S, Haubeck HD, et al.. Modification of the in vitro replication of the human immunodeficiency virus HIV-1 TPSg, a polysaccharide fraction isolated from the Cupressaceae Thuja occidentalis L arborvitae. Haematol Blood Transfus1992; 35:140-9. https://doi.org/10.1007/978-3-642-76829-3_24

98. Halder S, Bharal N, Mediratta PK, Kaur I, Sharma KK. Antiinflammatory, immunomodulatory and antinociceptive activity of Terminalia arjuna Roxb bark powder in mice and rats. Indian ExpBiol 2009; 47:577-83.

99. Sinha K, Mishra NP, Singh J, Kanjua SPS. Tinospora cordifolia, a reservoir plant for therapeutic applications: A review. Indian J TraditKnowl 2004; 3:257-70.

100. Samir P, David B, Otilia JF, Patel MM, Shah KK. Scrutinizing the role of aqueous extract of Trapa bispinosaas an immunomodulator in experimental animals. Int J Res Pharm Sci 2010; 1:13-9.

101. Agarwal S, Khadase S, Talele G. Bioactive immunomodulatory fraction from Tridaxprocumbens. Asian J Biol Sci 2010; 3:120-7. https://doi.org/10.3923/ajbs.2010.120.127

102. Rinku M, Prasanth VV, Parthasarathy G. Immunomodulatory activity of the methanolic extract of Urenalobata Linn. Int J Pharmacol 2008; 7:14. https://doi.org/10.5580/ba4

103. Davis L, Kuttan G. Immunomodulatory activity of Withania somnifera. J Ethnopharmacol 2000; 71:193-200. https://doi.org/10.1016/S0378-8741(99)00206-8

104. Devasagayam TPA, Sainis KB. Immune system and antioxidants, especially those derived from indian medicinal plants. Indian J Exp Biol 2002; 40:639-655

105. Gulati K, Ray A, Debnath PK. Immunomodulatory Indian Medicinal Plants. J Natural Rem 2002; 2(2):121-131

106. Sagarwat H, Md Khan Y. Immunomodulatory plants: A Phytoparmacological review. Pharmacog Rev 2007; 1(2):248260

107. Mukherjee PK, Nema NK, Bhadra S. Immunomodulatory leads from medicinal plants. Indian J Knowledge. 2014; 13(2):235-256 https://doi.org/10.1016/B978-0-12-397153-1.00022-6 